Article

\title{
Matching Score Models for Hyperspectral Range Analysis to Improve Wood Log Traceability by Fingerprint Methods
}

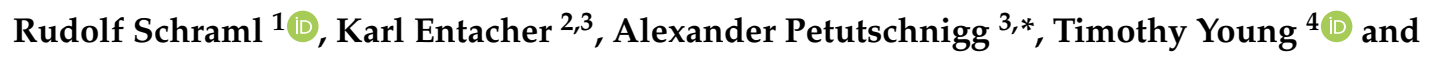 \\ Andreas Uhl ${ }^{1}$ \\ 1 Department of Computer Sciences, University of Salzburg, 5020 Salzburg, Austria; \\ rudi.schraml@gmail.com (R.S.); uhl@cosy.sbg.ac.at (A.U.) \\ 2 Holztechnikum Kuchl, 5431 Kuchl, Austria; karl.entacher@holztechnikum.at \\ 3 Department of Forest Products Technology and Timber Construction, University of Applied \\ Sciences Salzburg, 5412 Puch bei Hallein, Austria \\ 4 Center for Renewable Carbon, University of Tennessee, Knoxville, TN 37996, USA; tmyoung1@utk.edu \\ * Correspondence: alexander.petutschnigg@fh-salzburg.ac.at
}

Received: 8 May 2020; Accepted: 14 June 2020; Published: 2 July 2020

\begin{abstract}
Traceability of natural resources, from the cradle to the final product is a crucial issue to secure sustainable material usage as well as to optimize and control processes over the whole supply chain. In the forest products industries the material can be tracked by different technologies, but for the first step of material flow, from the forest to the industry, no systematic and complete technology has been developed. On the way to close this data gap the fingerprint technology for wooden logs looks promising. It uses inherent properties of a wood stem for identification. In this paper hyperspectral cameras are applied to gain images of Norway spruce (Picea abies [L.] Karst.) $\log$ end faces in different spectral ranges. The images are converted to a biometric template of feature vectors and a matching algorithm is used to evaluate if the biometric templates are similar or not. Based on this, matching scores specific spectral ranges which contain information to distinguish between different log end faces are identified. The method developed in this paper is a necessary and successful step to define scanning system parameters for fingerprint recognition systems for wood $\log$ traceability from the forest.
\end{abstract}

Keywords: wood traceability; biometric identification; fingerprint detection, hyperspectral imaging

\section{Introduction}

Traceability of wooden logs is a recent topic of research, as customers are getting more interested in the origin of their products. This led to certificates like the one of the Forest Stewardship Council [1] or the Program for the Endorsement of Forest Certification [2] that are documenting the sustainable production of wood. Even more legal actions and agreements like the European timber regulation EUTR No. 9952010 [3] were developed. This was done to claim disclosure of the provenance of timber and timber products that are placed on the European market in order to impede deforestation and illegal trading of timber. The availability of unambiguous tracking methods for logs from forest to forest products industries will guarantee the database for process analysis and process optimization and can therefore be seen as a necessity for further development. This topic is in the view of recent research all over the world and even scientific hackathons are organized (e.g., the Evergreen innovation camp 2019 in Vienna [4]) to solve the question of traceability of wood from the forest to the related industry. Different methods of log tracking systems were investigated in the past and they reach from the application of barcodes up to the usage of radio frequency identification (RFID) transponders (e.g., [5] 
or [6]), but tracking systems are only used for relatively high priced lumber up to now, due to technical and economical shortcomings when these systems are applied for rather inexpensive mass round wood. In this paper the main approach is based on inherent properties of a log based on biometric log end face characteristics as shown in [7-10]. The usage of log characteristics for tracking logs is not a new idea, e.g., in [11] and [12] surface properties of wood logs were used for identification and in [13] and [14] additional measurements with tracheid effect and X-ray were used. These applications can be used successfully in the sawmill environment, but they are not applicable on the forest site. In other research it was found that the log end faces contain a lot of information that can be used for biometric purposes [15-17]. To be successful on the forest site the measurement equipment has to be simple and insensitive to surrounding mechanical and other physical disturbances. For these reasons in [18] the application of commercial action cameras, which can be used in harsh environments, to collect log end images in the forest were tested. It was shown that the collection of images was satisfying in the forest, but the log end faces changed significantly during transport. Dirt accumulation, mechanical damages and color changes on the log end surface occurred during transport conditions. Based on these findings the following assumption is derived.

It is assumed that a hyperspectral image which contains not only the visible spectra, but depending on the technology also parts of the ultraviolet (UV)-spectra and near infrared (NIR) spectra, will be able to improve the detection of significant parts of log end faces.

The usage of hyperspectral imaging technologies ranges from large scale imaging down to lab scale sensors used in food safety, pharmaceutial applications forensic, etc. (examples are shown in [19] or [20] ) and therefore is very common in use. One main tasks for the successful application of these technologies is the development and usage of sophisticated analysis technologies for the given data quantities. e.g., [21] give an overview of main tasks and methods for hyperspectral remote sensing data analysis and in [22] the concept of active learning methods to improve data analysis models are shown. These papers show the direction of actual developments in the field of hyperspectral image analysis.

For wooden log tracking these hyperspectral methods are not investigated up to now, but results of other research groups (e.g., [23,24] or [25]) let assume that hyperspectral images might overcome the problems of the conventional methods as well as the shortcoming of the research approaches shown above.

Following this assumption, it is from special interest which spectra contains different information compared to other spectra, or the other way around, which spectra contain the same information than others. This knowledge is crucial to reduce the technical efforts and expenses while collecting the data in the forest. Within this paper a method is shown, which is able to calculate distance measures between different spectral images of complex shaped wooden log end faces. This enables the investigation of spectral ranges which contain different information and are thus valuable to improve image acquisition for fingerprint methods in the future.

\section{Materials and Methods}

\subsection{Wood Samples}

Initially, 100 different Norway (Picea Abies) spruce logs logs ( $4.5 \mathrm{~m}$ length) were collected on 24 and 25 September2018 in a grove near Corcieux, France (48.1968 N; 6.8869 E). Since the logs were selected from piles, it is not possible to know which logs belong to the same tree. Norway spruce was chosen for this study as it is the main tree species in Austria. These logs were transported to Freiburg in Germany where they were used for further data acquisitions. For the experiments in this paper a wood disc was cut from the lower end of each log. The outside of each disc was sanded with a mesh width of 120 to reduce the influence of manual chainsaw cuts at scanning. This mesh width was chosen as it gave good results for the wet samples. To avoid surface cracks due to wood shrinkage 
and discoloration due to oxidation processes the slices were packed individually in plastic bags during transport and intermediate storage.

\subsection{Scanning System}

The samples were scanned at Stemmer Imaging in Munich by two pupils of the Holztechnikum Kuchl, which used two different multispectral line scanners. The first scanner was a so called Specim FX10, which scans the spectra between a wavelength from $445 \mathrm{~nm}$ to $983 \mathrm{~nm}$ with a bandwidth of approx. $3 \mathrm{~nm}$. The second scanner was a Specim FX17 which provides scans between $990 \mathrm{~nm}$ to 1665nm also with a bandwidth of approx. $3 \mathrm{~nm}$. Therefor the Specim FX10 uses mainly the visible light (VIS) spectra and the Specim FX17 uses mainly parts of the NIR spectra. For the scanning setup a resolution of $640 \times 640$ pixel was chosen. The scanning system is shown in Figure 1a-Halogen light was used for lighting. Each wood disc was scanned with both cameras. For this purpose, each disc was pushed through the system by hand and the speed was synchronized with a trigger. The hyperspectral data, i.e., the translation from line scanning data to a hyperspectral cube, was performed by the acquisition software Perception Studio.

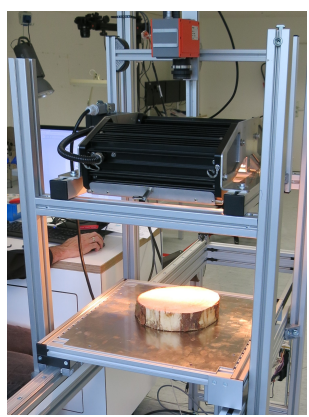

(a) Scanning system

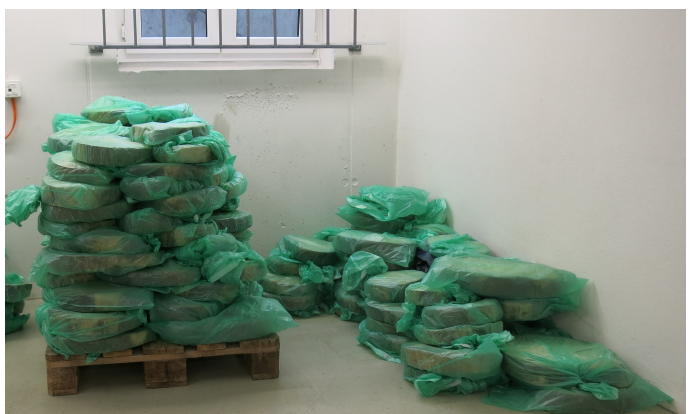

(b) Wood disc storage

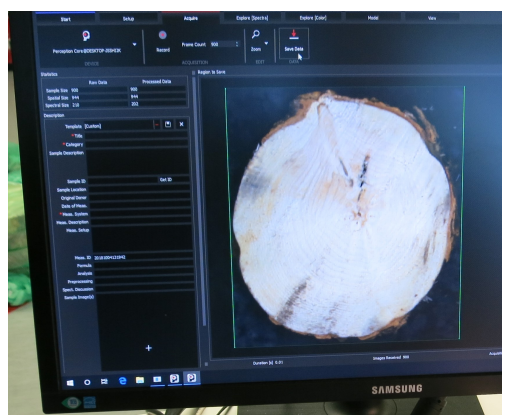

(c) Hyperspectral cube cropping

Figure 1. The sensor system shows the line scanner Specim FX10 which was mounted on a metal frame and the slice is moved perpendicular to the scanned line manually. The stored samples were closed airtight to avoid surface changes. Each hyperspectral cube was cropped to reduce the massive amount of data.

\section{Image Analysis}

The hyperspectral data (captured with the FX10 or FX17 camera) from each disc were stored in a special format (HSD-hyperspectral data) utilized by Perception Studio. A HSD file contains all spectral bands of a disc in kind of of a hyperspectral cube. It was necessary to convert the data to the ENVI format for which open source image processing libraries are available. Subsequently, for each hyperspectral cube the grayscale images (PNG) for each band were extracted and are denoted as cross-section (CS) images. In case of the FX10 this results in 202 CS-Images and for the FX17 in 196 CS-Images per wood disc. This conversion enables to apply standard image processing algorithms.

For each camera, CS-Images for selected bands of wood disc \#E001B are illustrated in Figure 2. It can be recognized that the width of the wood disc for the FX10 and FX17 images differs. We assume that this is caused by the acquisition software and an inaccurate trigger synchronization. In order to assess the similarity between different CS-Images, i.e., to compute distance measures between CS-Images, biometric features for each CS-Image are computed. Biometric features of a CS-Image are denoted as templates. Template computation is performed in two consecutive steps: CS-Registration and Feature Extraction which are described, subsequently. 


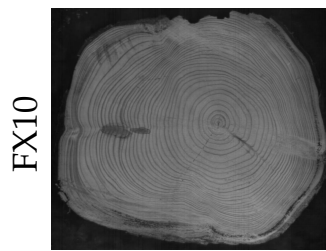

(a) $445 \mathrm{~nm}$

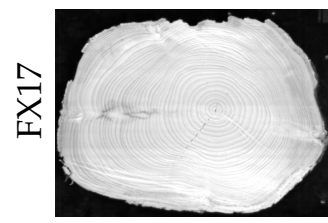

(f) $990 \mathrm{~nm}$

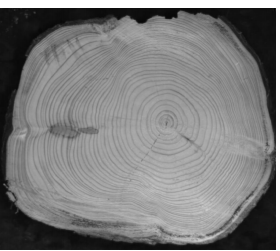

(b) $551 \mathrm{~nm}$

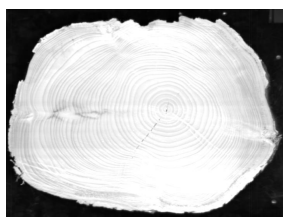

(g) $991 \mathrm{~nm}$

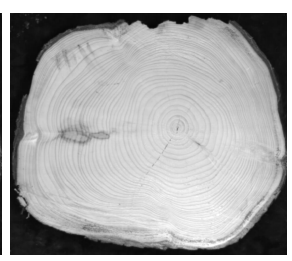

(c) $658 \mathrm{~nm}$

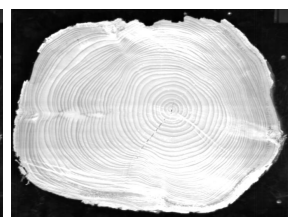

(h) $1270 \mathrm{~nm}$

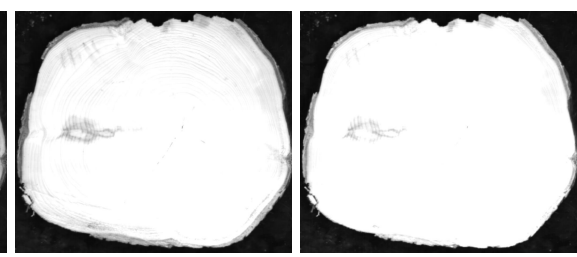

(d) $767 \mathrm{~nm}$

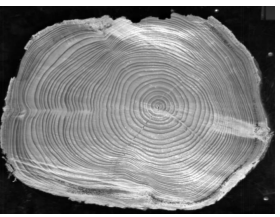

(i) $1410 \mathrm{~nm}$ (e) $878 \mathrm{~nm}$

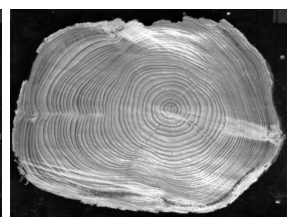

(j) $1553 \mathrm{~nm}$

Figure 2. Exemplary cross-section (CS)-Images from different hyperspectral bands of the wood disc \#E001B.

\subsection{CS-Registration}

Initially, the CS area in each CS-Image needs to be localized, i.e., segmented from the background. For this purpose, the feature-based segmentation approach by [26], which is based on a combination of superpixels and GraphCut regularization, was utilized. For our experiments we utilized the Python code (https: / / borda.github.io/pyImSegm/) provided by the authors. Color features were utilized and each CS-Image was segmented into two labels in order to segment fore- and background. Figure 3a,b show exemplary segmentation results for wood disc \#E001B for the first band captured with the FX10 and FX17, respectively. The superpixel clustering is based on Simple Linear Iterative Clustering (SLIC) for which we utilized a superpixel size of 40 pixel and for the regularization parameter a value of 0.25 . The segmentations are computed using the variant where the Gaussian Mixture Model (GMM) is created from a set of images, in our case from all CS-Images of a band and camera. Each segmentation result was further processed in order to remove holes and to select the largest area for which the concave hull was determined. The concave hull coordinates were stored for each CS-Image.

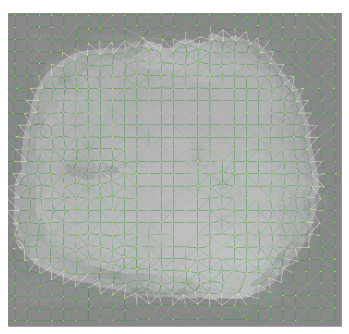

(a) FX10— \#E001B at $445 \mathrm{~nm}$

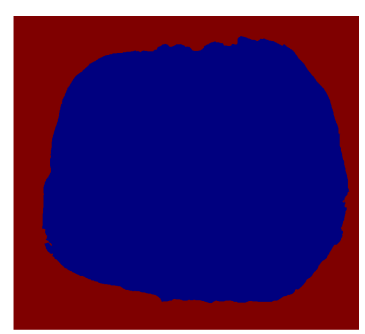

Figure 3. Unsupervised segmentation using superpixel, model estimation and GraphCut.

All computations which are described subsequently, were performed using Java code which was was developed in the context of the respective publications and extended for this work. For specific details, we refer to the referenced publications. Same as in previous works (e.g., [10]), biometric templates of CS-Images are computed in order to assess the similarity of CS-Images of a wood disc captured at different wavelengths. Therefore, the CS is registered to a unique position in terms of rotation and scale. The pith position serves as unique reference point and is determined using the approach proposed in [17]. Local orientation estimates and their intersections are utilized to determine a candidate for the pith position. Local orientation estimates are computed in the segmented CS area. In Figure 4, the pith estimation results for wood disc \#E001B captured with the spectral camera FX10 and F17 are shown in the first and second row, respectively. Furthermore, the center of mass (CM) is computed and the pith position to $\mathrm{CM}$ vector is used to rotationally align the CS to the horizontal axis 
as shown in images of the first and second column of Figure 4. Rotated images are scaled to a width of 400 pixels which results in a registered CS-Image. Consequently, for all registered CS-Images the pith to CM boundary vector has a fixed length of 200 pixels. This differs to the width utilized in our previous works due to the lower image resolution provided by the spectral cameras. Furthermore, our feature extraction approach is based on a circular grid as described in the next section.
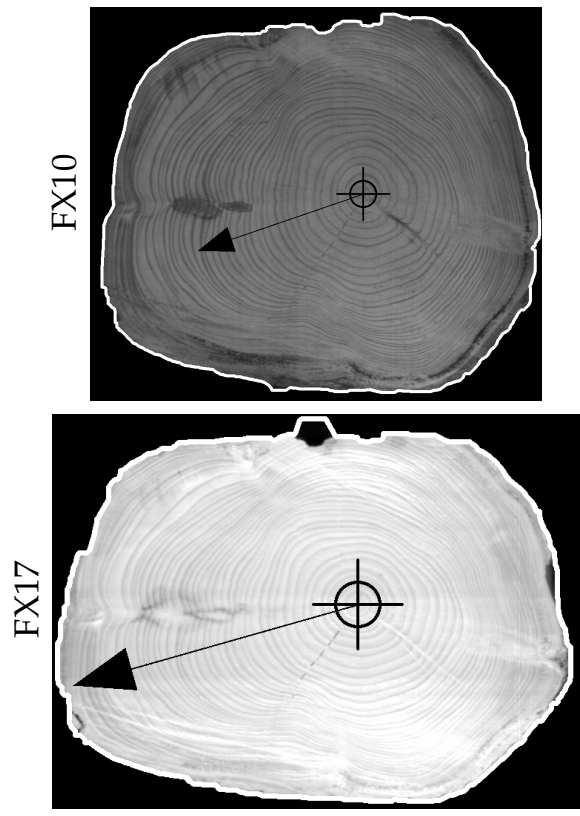

(a) Pith Estimation/Segmentation
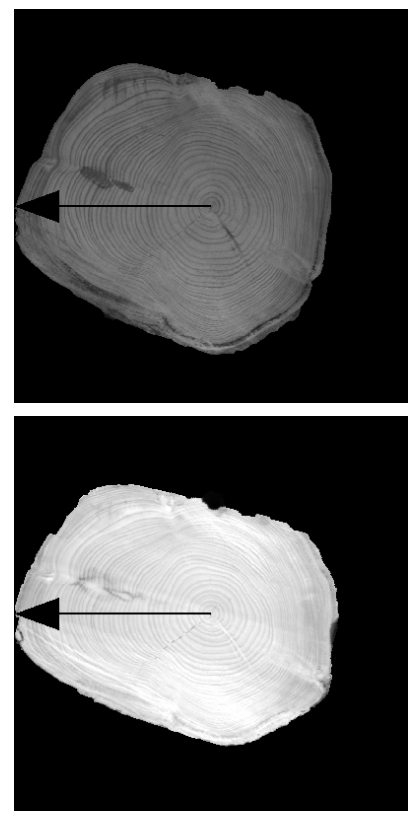

(b) Registrated CS
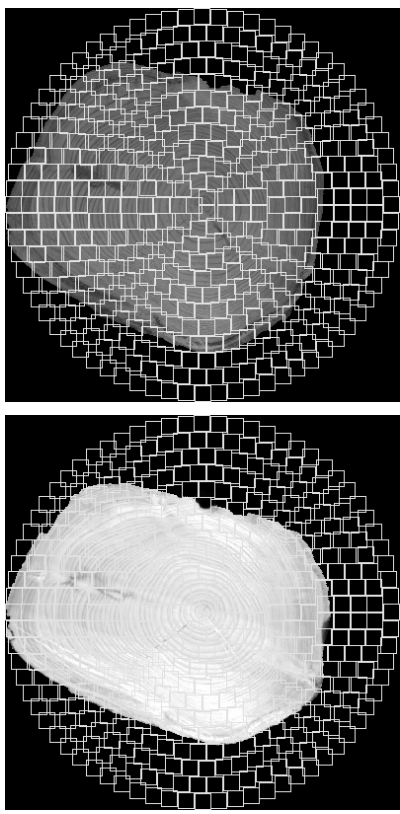

(c) Circular grid

Figure 4. Illustration of the feature extraction pipeline for wood disc \#E001B for the first band of both cameras.

\subsection{Feature Extraction}

As in [10], the texture-feature based approach by [27] is adopted and extended to compute and compare CS-Codes from CS-Images. Thus, the Gabor filterbank is extended to six different filters based on the following parameters: $G(\lambda, \theta, \sigma, \gamma)=G(\lambda, \sigma)=$ $((2,1),(3.5,2),(4.5,3),(5.5,3),(6.5,3),(7.5,3)), \theta=\{0,22.5, \ldots, 135,157.5\}, \gamma=0.7 . \lambda$ is the filter wavelength, $\theta$ represents the orientation, $\sigma$ is the standard deviation and $\gamma$ specifies the filter aspect ratio. This filterbank enables to capture the annual ring pattern frequency and orientation in local regions.

For feature extraction, the Gabor filterbank is applied to the local image regions of the registrated CS-Image, predefined by the circular grid as shown in the images in the right column of Figure 4.

Only local image regions within the CS area are processed. For each local region the Gabor filterbank is applied which results in a filter response for each filter. For each filter response the standard deviation is computed which leads to a feature vector with 48 standard deviation values per local region. Finally, the biometric template of a CS-Image is composed by all feature vectors computed for the local regions in the circular grid. Feature vectors of local regions outside the CS are set to NULL. Note, that in previous works a rectangular grid was utilized. This should have less impact on the biometric templates but is suited to reduce the size of the biometric templates because a large amount of background region is ignored while the shape information still is part of the biometric template.

\subsection{Template Matching}

In [8], three different matching procedures have been introduced. Note, that for this work no rotation compensation in the matching procedure is required. There are no rotational variations to compensate for. The first matching procedure of [8] which is focused on annual ring pattern 
information is utilized. This procedure is denoted as an annual ring pattern matching score $\left(\mathrm{MS}_{A P}\right)$. The aim of the other two procedures is to include shape information and the results in [8] confirmed the positive impact in terms of discriminative power resulting in a higher recognition accuracy. We are mainly interested in changes of the textural information shown at different wavelengths and thus shape information is not valuable. $\mathrm{MS}_{A P}$ for two CS templates is computed by

$$
M S\left(C S_{1}, C S_{2}\right)=\frac{1}{M} \sum_{i=0}^{n} D\left(C S_{1}(i), C S_{2}(i)\right)
$$

where $C S_{1}, C S_{2}$ are two feature vectors of the CS-Codes which are compared, $i$ specifies the index of the local region. As distance function $(D)$ the L1 norm is utilized which outperformed the L2 norm in a previous work [28]. The distance function $\left(D_{A P}\right)$ ignores feature vector pairs which are not in the intersection of both CSs. In the current implementation this is the case if one of both feature vectors is set to NULL. For normalization, $M$ is defined by the amount of considered feature value pairs.

\section{Experiments and Results}

For the experimental evaluation for all spectral CS-Images of each wood disc, captured with FX10 or FX17, biometric templates were computed. For each wood disc and the captured spectral CS-Images with a camera, the segmentation result computed for the first band (FX10 $=444 \mathrm{~nm}, \mathrm{FX17}=990 \mathrm{~nm})$ and the group segmentation model is utilized for all other spectral CS-Images. In the exactly identical way the pith is estimated for the segmented CS-Image from the first band and utilized for the remaining.

This ensures that all CS-Images of a wood disc captured with a camera are registrated in the exact same way. This is crucial in order to avoid side affects caused by varying segmentation and pith estimation results. Furthermore, for some bands CS segmentation is very difficult and pith estimation is impossible due to the absence of a clear annual ring pattern (see Figure 2). For feature extraction, a local region size of 16 pixel was utilized and biometric templates were computed for all CS-Images.

Matching scores were computed for all pairs of biometric templates from each wood disc captured with a camera. In human biometrics scores computed between templates of one individual are are referred to as intraclass scores. Interclass scores are computed between templates from different individuals. For this work, we denote intraclass scores which are computed between templates from on individual but different bands as spectral scores. Interclass scores are computed between biometric templates from different wood discs for the same band.

Based on the spectral scores, the similarity and non-similarity of different spectral bands is analyzed. The interclass scores for each band enable to draw conclusions on the suitability of the different bands to discriminate between different wood discs. The overall mean spectral distances of all pairs of spectra for 100 wood discs of each camera are shown in the distance matrices of Figure 5 . This distance matrix is calculated for all CS's hence it can be seen if the spectral score between different spectra bands can be observed for all CS's or not.

The matrices in Figure 5 are symmetric and bright areas indicate that the feature vectors between two spectra bands are similar while dark areas indicate that feature vectors between two spectra bands are different. In Figure $5 \mathrm{a}, \mathrm{b}$ the results for one specific CS-image (\#E001B) are shown and compared to the mean values over all CS-images in Figure $5 \mathrm{c}$,d. Based on this matrix for the spectral camera FX10 (see Figure $5 \mathrm{c}$ ) two different spectral ranges are estimated by visual inspection. These are the spectral ranges from $450 \mathrm{~nm}$ to approx. $740 \mathrm{~nm}$ and from 740 to $950 \mathrm{~nm}$. These two ranges seem to show different features, as the feature vectors are different. For the spectral camera FX17 (see Figure 5d) it is estimated that there are three different spectral ranges (from $900 \mathrm{~nm}$ to $1150 \mathrm{~nm}$, from $1050 \mathrm{~nm}$ to $1360 \mathrm{~nm}$ and from $1360 \mathrm{~nm}$ to $1800 \mathrm{~nm}$ ) where the feature vectors show different values based on the matching score.

To get an impression about the usability of different spectral bands for log traceability the mean interclass scores per band for all CSs are shown in Figure 6. Note that no normalization has been applied. A high mean-interscore expresses that the matching scores between CS-Images from different 
wood discs are high and the wood discs are not similar to each other. A low mean-interscore shows that different wood discs are very similar to each other which is a problem for a biometric system.

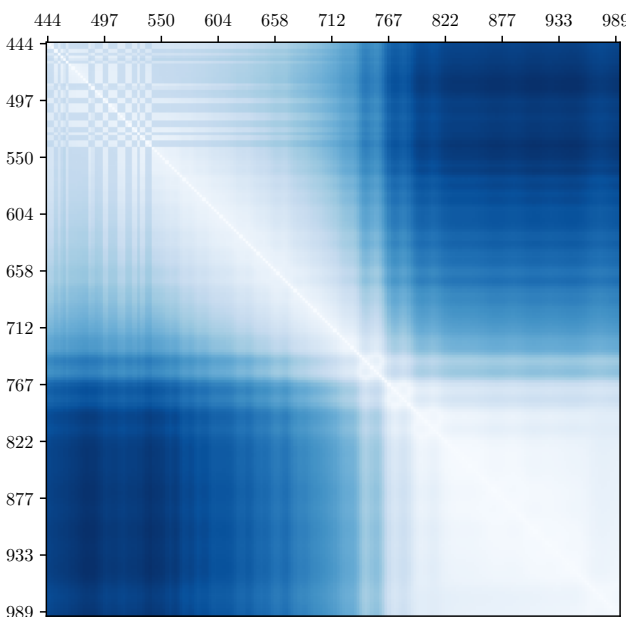

(a) FX10- \#E001B

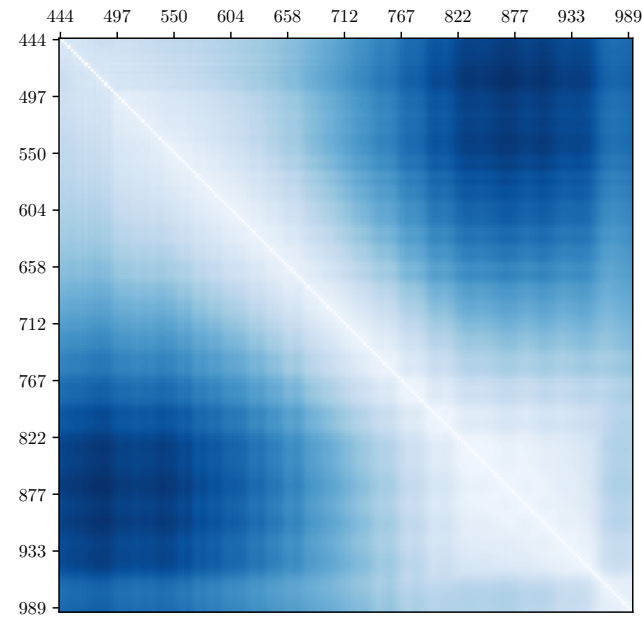

(c) FX10-Mean spectral distances

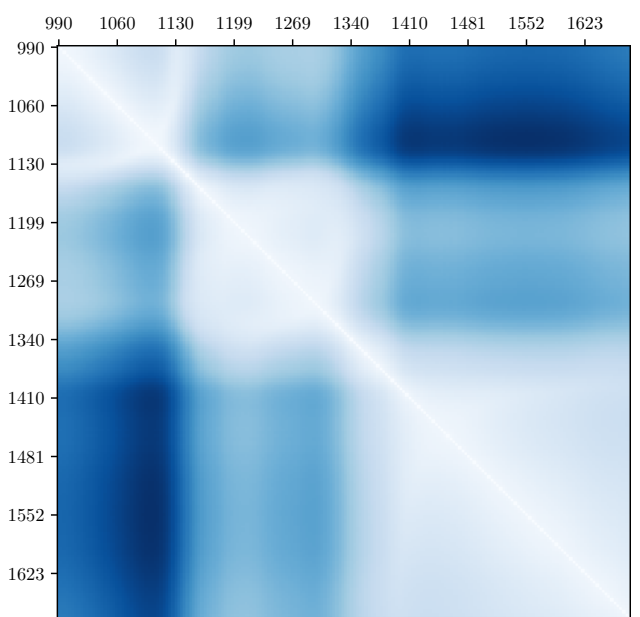

(b) FX17— \#E001B

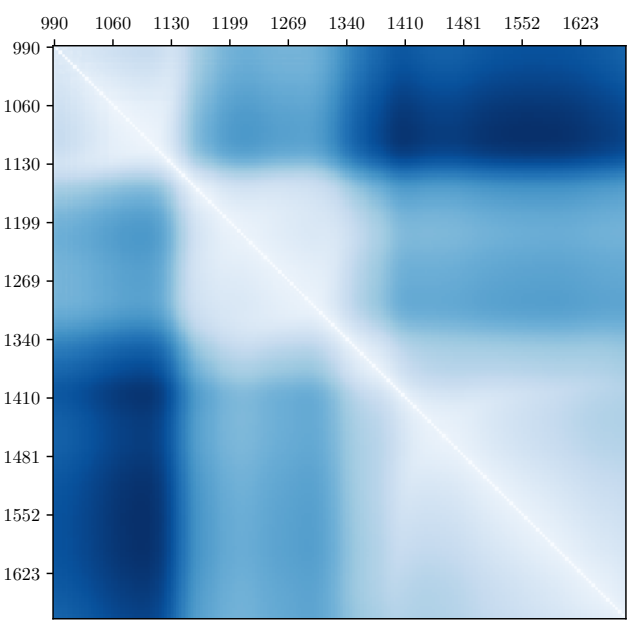

(d) FX17-Mean spectral distances

Figure 5. The distance matrices illustrate the spectral distances computed for all bands captured with the FX10 and FX17 hyperspectral camera. In the first row, the distance matrices for disc \#E001B are shown and in the second row the mean distance matrices for all 100 discs are illustrated.

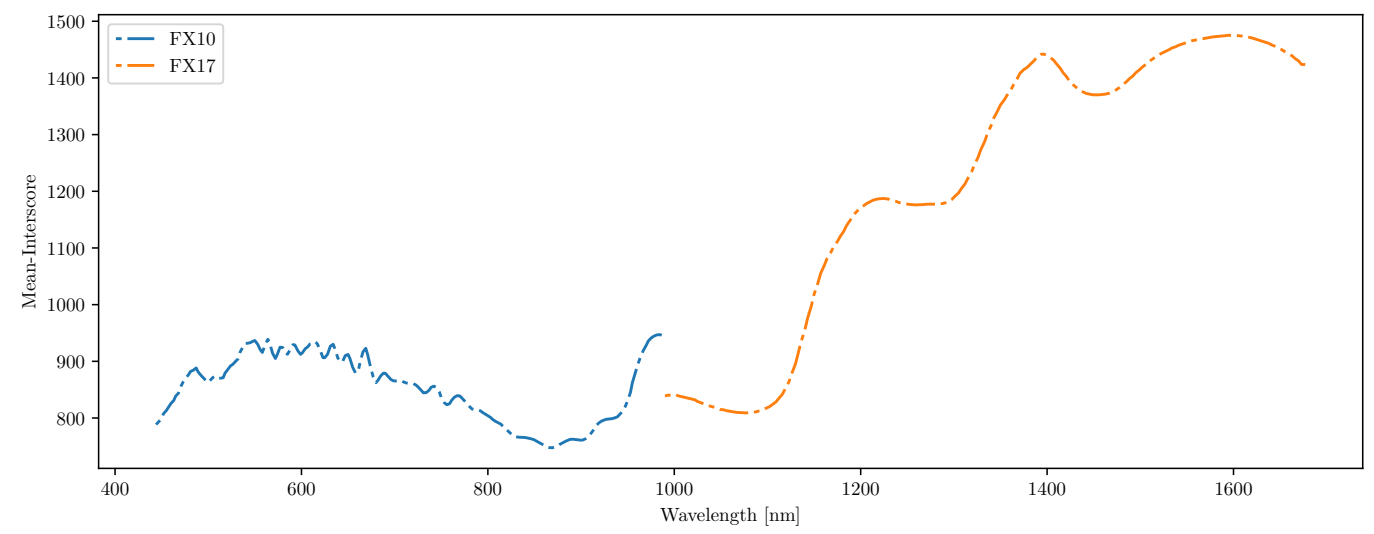

Figure 6. Mean interscores for all captured spectral bands. 
The higher the mean-interscore, the more discriminative are the CS-Images of the 100 discs in the chosen spectral band, i.e., if this value is high, this spectral band is good to identify different $\log$ end faces. Based on the interclass-scores in Figure 6, it seems that the NIR spectra $(>760 \mathrm{~nm})$ are much better for detecting different log end faces as the interclass scores are much higher for the spectral bands of spectral camera FX17 compared to the spectral camera FX10. Furthermore, approx. five local maxima can be expected out of the mean-interscore plot, two for the spectral camera FX10 and four for camera FX17 while one peak appears in the crossing spectra measured by camera FX10 and camera FX17 and is only counted as one. The wavelength at the peaks in the NIR spectra correspond to specific overtones of $\mathrm{OH}$-bond and $\mathrm{CH}$-bond vibration modes what allows a chemical interpretation of these observations.

\section{Conclusions}

The results of the spectral analysis showed that log end face images improved with hyperspectral camera systems in the spectral range between $400 \mathrm{~nm}$ and $1800 \mathrm{~nm}$ lead to different images. This supports the assumption that it is possible to gain additional information about one log end face if images in different spectral ranges are taken.

The fingerprint related method of feature extraction was applied successfully which led to specific feature vectors per image. These vectors are the basis to calculate pattern matching scores. The matching scores are used to investigate the similarity of spectral bands per log end face and the differences between the different log end faces per spectral band. The following observations were made for the investigated samples: In the spectral range between $450 \mathrm{~nm}$ and $1000 \mathrm{~nm}$ two intervals to gain different spectral images were observed and in the spectral range between $1000 \mathrm{~nm}$ and $1700 \mathrm{~nm}$ three intervals to gain different spectral images were observed. Based on this observation, it is assumed that up to 5 spectral bands (one of each spectral range) contain most of the information within the feature vectors. This assumption is supported by the results of the interscores between the $\log$ end faces per spectra. Here it can also be seen that the differentiation between logs takes local maxima in five different spectral band regions. There is evidence that the NIR spectra create a better distinction between different log end faces than the visible spectra. The objective of this study was the development of a methodological basis to use hyperspectral images for log tracking. The results are promising for the development of a system based on the methods described, but it is not possible to derive an overall valid model for Norway spruce wood (picea abies [L.] Karst.) or other wood species from this specific sample.

Nevertheless, the method shown is applicable to use hyperspectral imaging for different wood samples and species and we invite different research groups in log tracking all over the world to work on this approach to develop the basis for a globally accepted log tracking system, as wood trade is a global issue.

Further investigations should and will focus on following questions:

- More detailed analysis on the 'best' spectral bands for the camera system. It is assumed that cameras which works in two up to four different spectral bands will be applied in the future.

- Usage of wood log end faces from different locations and different wood species to be able to work in practical surroundings.

- Development and implementation of statistical process control methods to optimize the production processes between forest and forest product industries.

Summarizing the usage of hyperspectral images are promising to reach a tracking method for wood logs and the next steps will be from lab scale to field application. Therefore the technical equipment necessary has to be reduced and the analysis algorithms have to be improved. For both tasks the findings in this work are useful and existing results of other fields of research (e.g., algorithms from remote sensing) will be utilized in future research. 
Author Contributions: Conceptualization: A.P. and R.S.; methodology: A.P. and R.S.; software: R.S.; validation: A.P. and R.S.; formal analysis: A.P. and K.E.; data curation: R.S.; writing-original draft preparation: A.P., K.E. and R.S.; writing-review and editing: T.Y., A.U. and K.E.; visualization: R.S. All authors have read and agreed to the published version of the manuscript.

Funding: This research was funded by the Austrian Science Fund (FWF) under the grant number I 3653.

Acknowledgments: The authors thank the support of open access funding by the Austrian Science Fund (FWF).

Conflicts of Interest: The authors declare no conflict of interest.

\section{Abbreviations}

The following abbreviations are used in this manuscript:

$\begin{array}{ll}\text { CS } & \text { Cross-section } \\ \text { UV } & \text { Ultra-violet } \\ \text { VIS } & \text { Visible-light } \\ \text { NIR } & \text { Near-infrared } \\ \text { MS } & \text { Matching score } \\ \text { CM } & \text { Center of Mass }\end{array}$

\section{References}

1. FSC. Homepage of the Forest Stewardship Council. Available online: www.fsc.org (accessed on 21 April 2020).

2. PEFC. Homepage of the Programme for the Endorsement of Forest Certification. Available online: www. pefc.at (accessed on 21 April 2020).

3. EuropeanParliament. Regulation (EU) No 995/2010 of the European Parliament and of the Council of 20th October 2010 Laying Down the Obligations of Operators Who Place Timber and Timber Products on the Market; EuropeanParliament: Brussels, Belgium, 2010.

4. Evergreen. Homepage of the Evergreen Innovation Camp 2019 in Vienna. Available online: www.evergreeninnovationcamp.io (accessed on 21 April 2020).

5. Tzoulis, I.; Andreopoulou, Z. Emerging Traceability Technologies as a Tool for Quality Wood Trade. Procedia Technol. 2013, 8, 606-611. [CrossRef]

6. Björk, A.; Erlandsson, M.; Häkli, J.; Jaakkola, K.; Nilsson, Å.; Nummila, K.; Puntanen, V.; Sirkka, A. Monitoring environmental performance of the forestry supply chain using RFID. Comput. Ind. 2011, 62, 830-841. [CrossRef]

7. Schraml, R.; Petutschnigg, A.; Uhl, A. Validation and Reliability of the Discriminative Power of Geometric Wood Log End Features. In Proceedings of the IEEE International Conference on Image Processing (ICIP'15), Quebec City, QC, Canada, 27-30 September 2015. [CrossRef]

8. Schraml, R.; Hofbauer, H.; Petutschnigg, A.; Uhl, A. Tree Log Identification Based on Digital Cross-Section Images of Log Ends Using Fingerprint and Iris Recognition Methods. In Proceedings of the 16th International Conference on Computer Analysis of Images and Patterns (CAIP'15), Valletta, Malta, 2-4 September 2015, Springer: Berlin, Germany, 2015; pp. 752-765. [CrossRef]

9. Schraml, R.; Charwat-Pessler, J.; Petutschnigg, A.; Uhl, A. Towards the applicability of biometric wood log traceability using digital log end images. Comput. Electron. Agric. 2015, 119, 112-122. [CrossRef]

10. Schraml, R.; Hofbauer, H.; Petutschnigg, A.; Uhl, A. On rotational pre-alignment for tree log end identification using methods inspired by fingerprint and iris recognition. Mach. Vis. Appl. 2016, 27, 1289-1298. [CrossRef]

11. Chiorescu, S.; Grönlund, A. The Fingerprint approach: using data generated by a 2-axis log scanner to accomplish traceability in the sawmill's log yard. For. Prod. J. 2003, 53, 78-86.

12. Chiorescu, S.; Grönlund, A. The Fingerprint Method: Using Over-bark and Under-bark Log Measurement Data Generated by Three-dimensional Log Scanners in Combination with Radiofrequency Identification Tags to Achieve Traceability in the Log Yard at the Sawmill. Scand. J. For. Res. 2004, 19, 374-383. [CrossRef]

13. Flodin, J.; Oja, J.; Grönlund, A. Fingerprint traceability of logs using the outer shape and the tracheid effect. For. Prod. J. 2008, 58, 21-27. 
14. Flodin, J.; Oja, J.; Grönlund, J. Fingerprint traceability of sawn products using industrial measurement systems for x-ray log scanning and sawn timber surface scanning. For. Prod. J. 2008, 58, 11.

15. Longuetaud, F.; Mothe, F.; Kerautret, B.; Krähenbühl, A.; Hory, L.; Leban, J.M.; Debled-Rennesson, I. Automatic knot detection and measurements from X-ray CT images of wood: A review and validation of an improved algorithm on softwood samples. Comput. Electron. Agric. 2012, 85, 77-89. [CrossRef]

16. Breinig, L.; BrüChert, F.; Baumgartner, R.; Sauter, U.H. Measurement of knot width in CT images of Norway spruce (Picea abies [L.] Karst.)-Evaluating the accuracy of an image analysis method. Comput. Electron. Agric. 2012, 85, 149-156. [CrossRef]

17. Schraml, R.; Uhl, A. Pith Estimation on Rough Log End Images using Local Fourier Spectrum Analysis. Proceedings of the 14th Conference on Computer Graphics and Imaging (CGIM'13), Innsbruck, Austria, 12-14 February 2013. [CrossRef]

18. Charwat-Pessler, J.; Schraml, R.; Entacher, K.; Petutschnigg, A. Tracking logs with RGB images within the wood supply chain: a preliminary study on image acquisition. For. Prod. J. 2015, 66, 176-184.. [CrossRef]

19. Camps-Valls, G.; Tuia, D.; Gómez-Chova, L.; Jiménez, S.; Malo, J. Remote Sensing Image Processing; Morgan and Claypool: San Rafael, CA, USA, 2011.

20. Richards, J.A.; Jia, X. Remote Sensing Digital Image Analysis: An Introduction; Springer: New York, NY, USA; Berlin/Heidelberg, Germany, 2006.

21. Bioucas-Dias, J.; Plaza, A.; Camps-Valls, G.; Scheunders, P.; Nasrabadi, N.; Chanussot, J. Hyperspectral Remote Sensing Data Analysis and Future Challenges. IEEE Geosci. Remote Sens. Mag. 2013, 1, 6-36. [CrossRef]

22. Svendsen, D.H.; Martino, L.; Camps-Valls, G. Active emulation of computer codes with Gaussian processes-Application to remote sensing. Pattern Recognit. 2020, 100, 107103. [CrossRef]

23. Ruano, A.; Zitek, A.; Hinterstoisser, B.; Hermoso, E. NIR hyperspectral imaging (NIR-HI) and $\mu$ XRD for determination of the transition between juvenile and mature wood of Pinus sylvestris L. Holzforschung 2019, 73, 621-627. [CrossRef]

24. Chen, J.; Li, G. Prediction of moisture content of wood using Modified Random Frog and Vis-NIR hyperspectral imaging. Infrared Phys. Technol. 2020, 105, 103225. [CrossRef]

25. Ma, T.; Inagaki, T.; Ban, M.; Tsuchikawa, S. Rapid identification of wood species by near-infrared spatially resolved spectroscopy (NIR-SRS) based on hyperspectral imaging (HSI). Holzforschung 2019, 73, 323-330. [CrossRef]

26. Borovec, J.; Švihlík, J.; Kybic, J.; M.D., D.H. Supervised and unsupervised segmentation using superpixels, model estimation, and graph cut. J. Electron. Imaging 2017, 26, 1-17. [CrossRef]

27. Jain, A.; Ross, A.; Prabhakar, S. Fingerprint matching using minutiae and texture features. In Proceedings of the International Conference on Image Processing (ICIP'01), Thessaloniki, Greece, 7-10 October 2001; Volume 3, pp. 282-285.

28. Schraml, R.; Charwat-Pessler, J.; Uhl, A. Temporal and longitudinal variances in wood log cross-section image analysis. In Proceedings of the IEEE International Conference on Image Processing (ICIP'14), Paris, France, 27-30 October 2014. [CrossRef]

(C) 2020 by the authors. Licensee MDPI, Basel, Switzerland. This article is an open access article distributed under the terms and conditions of the Creative Commons Attribution (CC BY) license (http://creativecommons.org/licenses/by/4.0/). 